\title{
La posibilidad del Estado ideal de Platón en la República y en las Leyes. Una alternativa a la interpretación de André Laks de la filosofía política de Platón
}

\author{
ULRIKE BRUCHMÜLLER \\ Instituto de Investigaciones Filológicas \\ Universidad Nacional Autónoma de México \\ u.bruchmueller@yahoo.de
}

Resumen: La posibilidad del Estado ideal de la República se funda dialécticamente en la Idea del Bien como causa de todo ser. El Estado como hipótesis ( a la unidad de las Ideas, garantizada por la Idea del Bien. Por otro lado, el Estado en las Leyes no se reduce a la unidad de las Ideas, sino sólo a la unidad del alma. Es el segundo mejor de los Estados, pero el primero, contra la tesis fundamental de Laks, también es posible para los hombres.

Palabras clave: filosofía de la historia, método hipotético, dialéctica, doctrina del alma

\begin{abstract}
The possibility of the ideal state in the Republic is grounded dialectically on the Idea of the Good as the cause of all being. The state as hypothesis

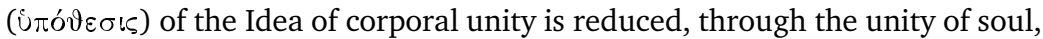
to the unity of Ideas, guaranteed by the first principle. The state of the Laws, on the other hand, is not reduced to the unity of Ideas, but only to the unity of soul. It is the second-best state after the ideal state which is -in contrast to the thesis of Laks- also possible for men.
\end{abstract}

Key words: philosophy of history, hypothetical method, dialectic, doctrine of the soul

En su libro La filosofía política de Platón a la luz de las Leyes, André Laks intenta reconstruir a partir de las Leyes una historia del desarrollo de la filosofía política de Platón. Puesto que este enfoque parte de la premisa de que Platón no pudo haber alcanzado más que en su última obra la solución más elevada para su modelo del Estado ideal, desatiende el hecho de que los escritos de Platón no son autárquicos, pues, por el contrario, según Fedro 278b7-d7, el filósofo debe ser capaz de ayudar a su discurso; y, según el Político, ningún diálogo puede entenderse sin los demás; ${ }^{1}$ antes bien, el contenido de los diálogos, según Fedro

${ }^{1}$ En Político 285c4-287b2, el arte dialéctico que se practica en los diálogos se compara con el aprendizaje de la lectura, que no se practica con una sola palabra, sino con varias. 
269d2-272a3, está consensuado en todo momento en relación con las exigencias psíquicas de uno o varios receptores determinados. ${ }^{2}$ Ahora bien, los interlocutores en las Leyes, Clinias y Megilo, se caracterizan por su procedencia espartana (esto es, timocrática) y, por su decidido interés práctico, como extremadamente no filosóficos. Si Platón quería exponer aquí sus últimos puntos de vista, ¿no habría podido elegir interlocutores más apropiados? ${ }^{3}$ A continuación comentaré las tesis más importantes del libro de Laks que parecen apoyar una interpretación histórico-evolutiva.

En el primer capítulo, Laks defiende el punto de vista de que en el Estado platónico positivamente ordenado, sea el de la República, el del Político o el de las Leyes, no habría habido lugar para un personaje como Sócrates, puesto que él tenía meramente el cometido de combatir las falsas opiniones mediante la mayéutica y la refutación. La idea de que el Sócrates de los diálogos tempranos se diferencia sustancialmente de la elaborada figura del dialéctico platónico perfeccionado es un prejuicio común, pero lo ha contradicho la investigación. ${ }^{4}$ Antes bien, a los bosquejos constructivos del Estado en la República y en el Político los precede un diálogo aporético. En el libro primero de la República, el diálogo aporético elimina ejemplarmente las falsas opiniones de la

${ }^{2}$ En este artículo sigo la lectura de la escuela de Tubinga, que remite a la doc-

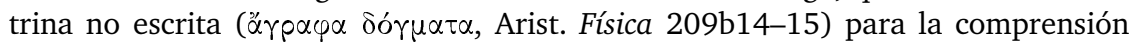
última de los diálogos (cfr., sobre todo, Krämer 1959; Gaiser 1998, y Reale 1997). Para una fundamentación hermeneútica de esta línea exegética, cfr. Szlezák 1985 y 2004. En oposición a esta lectura está la línea exegética histórico-evolutiva ( $c f r$., p.ej., Hermann 1839; Guthrie 1975, y Vlastos 1991), que no puede explicar las diferencias supuestas de los diálogos sino con una falta del autor, y, por otro lado, las interpretaciones unitarias que rechazan el recurso a la doctrina no escrita y están a favor de una interpretación pedagógica o proléptica (cfr., p.ej., Schleiermacher 1804, y Kahn 1996) si bien no pueden ofrecer una explicación completa y unitaria de todos los contenidos filosóficos de los diálogos. En un análisis bastante riguroso e impresionante, pero sin comprensión del significado metafísico de la filosofía platónica, Cherniss $(1944,1945)$ rechazó el informe de Aristóteles sobre las doctrinas no escritas de Platón.

${ }^{3}$ Cfr. Görgemanns 1960; Schöpsdau 1994, p. 126, y Szlezák 2004, pp. 44-53.

${ }^{4}$ Cfr. Szlezák 1985 y 2004. El diálogo que introduce a Sócrates como mayéutico muestra especialmente que el mismo Sócrates puede parir mejor que todos los presentes, de modo que la ironía socrática que está detrás de la metáfora de la mayéutica en el de Teeteto debería ser inconfundible (cfr. Szlezák 2004, 91-127).

Diánoia, vol. LIV, no. 63 (noviembre 2009). 
multitud sobre la justicia. ${ }^{5}$ De manera semejante, a los diálogos constructivos sobre la érı después en el Filósofo, se ha antepuesto el diálogo aporético Teeteto, que trata acerca del mismo concepto. También en los diálogos tardíos la purificación es una condición esencial de la dialéctica platónica (Sofista 226b1-231b2). Su importancia en el mejor de los Estados depende del grado de impureza en el que se encuentren las almas de los cuidadanos. Dado que las Leyes bosquejan sólo el segundo mejor de los Estados, la purificación necesariamente desempeña aquí un papel más importante que en el Estado ideal de la República. Así, también se recomienda ahí la lectura de los diálogos platónicos en la enseñanza escolar (Leyes 811b8-e5). Por el contrario, en la República sólo en unos pocos habita una mala naturaleza (500a5-7). A éstos, incluso antes de llegar a ser integrados en el Estado ideal, debe llevárseles a que, por vergüenza, confiesen que se han apaciguado y han quedado convencidos (501c4502a3). Apenas puede pasarse por alto la alusión al diálogo aporético con Trasímaco, en especial porque su persona dio lugar a esta discusión $(498 \mathrm{c} 5-\mathrm{d} 4){ }^{6}$

\section{II}

Además, Platón exige que en una buena constitución haya sabiduría en los gobernantes y consentimiento en los gobernados. Esta "doble normatividad" habría de ser, según Laks (p. 38), "el problema fundamental de la politeia platónica”, que Platón pudo haber percibido por primera vez en las Leyes.

La República, por el contrario, encubriría el problema a través de una doble definición de templanza (tanto en el sentido de la armonía como en el de la obediencia). Además, la unidad del Estado ${ }^{7}$ sólo concerniría a las dos clases superiores (465b8-10), pues los productores por naturaleza habrían de tener apetitos insaciables. De igual forma, el mito fenicio indicaría el empleo de la violencia contra los productores. En la República faltaría, entonces, casi por completo una mediación peda-

${ }^{5}$ No es posible encontrar fundamento alguno para la suposición de una redacción separada del libro primero bajo el título de Trasímaco (cfr. Szlezák 1984, y Kahn 1993).

${ }^{6}$ Para mayores detalles a este respecto, véase más adelante la p. 186.

${ }^{7}$ La objeción en Arist. Pol. 1261 b32 ss., a la que se une Laks (p. 41), en el sentido de que la unidad no sería realizable porque los vigilantes sin propiedad privada serían indiferentes al bien común, es insostenible, pues este bien común es mucho más valioso que cualquier bien individual (465d2-466d5). 
gógica entre los estamentos. Al sustituir en el Político el binomio entre gobernantes y productores con una diferenciación universal de caracteres valientes y templados, entre quienes se armonizarán las opiniones verdaderas sobre lo Bello, lo Justo y lo Bueno mediadas por la ley, se habría preparado el camino a la función pedagógica del preámbulo en las Leyes. No obstante, el empleo diverso de la metáfora del médico (Político 293a9-b8, Leyes 720a6-e4) muestra, según Laks, que no es sino en las Leyes donde se manifiesta un afán por alcanzar un verdadero consentimiento.

Efectivamente, la antinomia entre el saber filosófico y la naturaleza humana determina la construcción de la República. Los estudios de filosofía de la historia de Konrad Gaiser han mostrado que, en el desarrollo de la historia, Platón supuso dos tendencias en direcciones contrarias. El despliegue continuo y creciente de los deseos se compensa aquí mediante conquistas científicas cada vez mayores hasta la contemplación de la Idea del Bien como principio último. Mientras los hombres de la polis sana vivían según principios justos, en el transcurso del desarrollo se multiplicaban sus deseos. Esta tendencia negativa se subsana en los libros II y III mediante una educación organizada por el Estado (Gaiser 1998, pp. 226 s.). En el Estado purificado se alcanza el consentimiento mediante mentiras curativas, como es el caso, por ejemplo, del mito fenicio. ${ }^{8}$ En el libro V se pone de manifiesto que Sócrates no quería en absoluto introducir el verdadero Estado, pues temía la incredulidad de

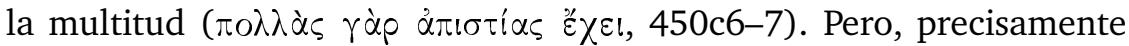
de parte de ella, representada aquí por los otros interlocutores, recibe la petición insistente de hacerlo (449a1-451b8). El empleo de la metáfora de las olas indica, entonces, la dificultad de persuadir a la multitud de aceptar el Estado ideal, pero al mismo tiempo muestra que esto es el propósito principal de los libros intermedios. Una y otra vez se destaca la naturaleza humana corrompida por los sofistas, la cual, no obstante, es vencida por un saber filosófico de rango cada vez más alto. Lo que en un principio parecía difícil es al final posible. Tal como la educación en los libros II y III ha superado la codicia (pleonexia) del Estado opulento, así la perfecta educación dialéctica supera la sofística: no mediante la violencia, sino con los medios de la retórica dialéctica. El enorme forcejeo de estas dos tendencias con la muy tardía, escasa, pero finalmente definitiva victoria de la filosofía - forcejeo que Laks percibe como una falta de conciencia de los problemas- se muestra,

\footnotetext{
${ }^{8}$ El uso de la violencia en el Estado purificado (415d8-e2) es un caso particular

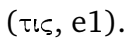


entonces, como un medio expresivo consciente del autor. La naturaleza humana tan fuertemente corrompida es purificada lentamente, pero con seguridad, mediante la educación filosófica y se la reconduce a su naturaleza verdadera. ${ }^{9}$

Además, en la República tampoco se opera con una doble definición de templanza, sino que, mediante la obediencia frente al mejor (al final frente al principio de unidad), se produce la armonía como imagen del Uno. La misma definición subyace también, por ejemplo, en Leyes 644b6-645c1.

Por lo demás, es necesario que la unidad de las clases superiores garantice la unidad del Estado entero (465b8-10), pues la clase social superior tiene en sus manos la educación de la clase inferior. Y a la inversa, también atrae al pueblo por su propia virtud. Según el mismo principio, el consentimiento de la multitud al gobierno de los filósofos se sigue casi automáticamente de la descripción de la naturaleza filosófica (498c5-502a4). Además, basta simplemente con comentar la educación del gobernante filósofo, puesto que de allí resulta la educación intelectual de la multitud. Por lo demás, de igual forma se pierde la unidad del Estado si la clase superior se divide (545c8-d4). ${ }^{10} \mathrm{Al}$ contrario de lo que piensa Laks, no hay en ello una contradicción.

La misma teoría de la historia se halla también en el Político, a saber, aquella según la cual, mediante su conocimiento perfecto de las Ideas hasta la fundamentación en los principios, ${ }^{11}$ el verdadero hombre de Estado imita adecuadamente el antiguo gobierno del dios (303b3-5) ejercido en tiempos prehistóricos (268d5-274e3) y, por lo tanto, subsana completamente la corrupción de la sociedad introducida durante la ausencia del dios (cfr. Gaiser 1998, pp. 210 s.; Bruchmüller 2008, pp. 18-53, 65-73, 176-181). Puesto que en el Político, al contrario de como se hace en la República, el hombre de Estado es colocado incluso

\footnotetext{
${ }^{9}$ Un análisis exacto de los libros intermedios se halla más adelante, en las pp. 181-189.

${ }^{10}$ Con respecto a la relación entre las dos clases, véanse también las pp. 185-187, infra.

${ }^{11}$ La condición necesaria para la existencia del hombre de Estado es la prueba de que "el más y el menos son mensurables, no sólo en su relación recíproca, sino

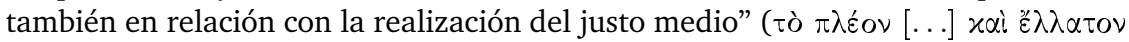

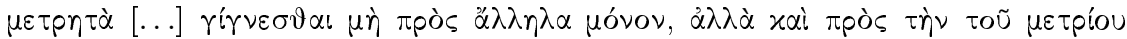

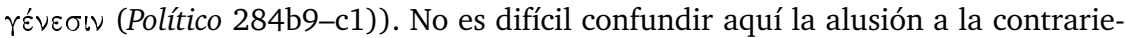
dad de los principios del Uno y de la Dualidad Indeterminada (cfr. Krämer 1959, pp. 155-163), cuyo tratamiento se omite por razones de la crítica a la escritura. En un pasaje posterior se indica otra vez el principio positivo con la expresión "un

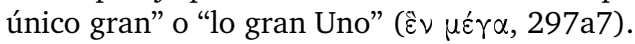

Diánoia, vol. LIV, no. 63 (noviembre 2009). 
por encima de toda ley, ese diálogo no representa un paso intermedio hacia las Leyes, sino que procede de una manera mucho más radical que la República. Por otra parte, a través de la diferenciación adicional de los caracteres de valiente y de templado no se eliminan las diferencias de clase; antes bien, es de suponer que la política educativa y matrimonial en el Político se refiera también sólo a las dos clases superiores, puesto que éstas, al igual que en la República, ${ }^{12}$ garantizan la unidad del todo, y que la valentía y la vergüenza son virtudes características del segundo estamento pero no del tercero. Por lo demás, ya la República conoce la mediación entre la templanza y la valentía a través de un ánimo filosófico (Rep. 374d8-376c6), la cual subyace en la totalidad del plan educativo del estamento de los vigilantes, o sea, del segundo estamento, en los libros II y III, y desde el punto de vista de su contenido tendría que ser idéntico al programa insinuado en el Político, de modo que aquí seguramente Platón no creyó haber encontrado una solución mejor.

Sin duda, en comparación con la República y con el Político, las Leyes son el diálogo que se dirige a los interlocutores menos filosóficos, y que, de acuerdo con ello, hace a la naturaleza humana las máximas concesiones. A diferencia de los otros dos diálogos, el Estado no se funda tampoco a base del conocimiento de las Ideas, sino sólo del conocimiento de los principios en el ámbito anímico. ${ }^{13}$ Esta enorme diferencia ontológica ${ }^{14}$ entre las condiciones necesarias de los dos esbozos de Estado produce también, naturalmente, una educación peor de la clase inferior, de tal modo que el consentimiento llega a ser aquí, por primera vez, realmente un problema.

Por consiguiente y en resumidas cuentas, Laks no puede demostrar aquí desarrollo alguno del consentimiento.

III

Después de un minucioso análisis de todos los escritos político-estatales de Platón, André Laks (pp. 101-108) intenta determinar nuevamente la relación entre la República y las Leyes: no obstante que la República parte de un concepto débil de posibilidad, es decir, que con un acercamiento la posibilidad ya está dada (Rep. 472b3-473b3), en el tanscurso

\footnotetext{
${ }^{12}$ Véase la p. 179, supra.

${ }^{13}$ Con respecto al nous como principio del alma y del movimiento, cfr. Leyes 895c1-898d1.

${ }^{14} \mathrm{El}$ teorema del rey filósofo ya no exige en las Leyes a un filósofo verdadero, sino únicamente un vínculo de poder con phronesis y sophrosyne (Leyes 711e7-712a3).
} 
de su exposición llega a defender la posibilidad de una identidad entre modelo y realidad. Esta ambigüedad contenida en la obra misma pudo dar a Platón la posibilidad de fijar su Estado ideal, en la República, en retrospectiva como una utopía, aunque con carácter modélico. En correspondencia con la discrepancia que ve entre las dos obras, Laks fija la fecha de la composición de la República en 387 a.C., es decir, cuarenta años antes de la redacción de las Leyes. Esta datación, sin embargo, es muy cuestionable ( $c f r$. Thesleff 1982).

El malentendido que subyace en esta interpretación procede, en mi opinión, de una interpretación errónea muy difundida del concepto de posibilidad en Platón. Por esa razón deseo indicar, en primer lugar, en qué sentido era posible para Platón el Estado ideal en la República, y mostrar que este texto no admite ambigüedad alguna. Esto permitirá, después, una comprensión de las Leyes que no debe suponer ningún cambio de posición del autor, y por eso, desde el punto de vista del método, ésta debería ser la interpretación preferente.

\section{III.1. REPÚBLICA}

Ya desde las explicaciones acerca del razonamiento de la República quedó claro que la posibilidad del Estado ideal llegó a ser garantizada mediante la recurrencia progresiva a un saber filosófico de rango cada vez más alto. ${ }^{15}$ Esto también se confirma mediante un análisis más detenido.

Una vez que Sócrates ha determinado, al final del libro IV, la justicia en el Estado y en el individuo, procede a determinar las otras cuatro constituciones. Al inicio del libro V intervienen Polemarco y Adimanto y piden al filósofo que explique qué quería decir al afirmar que, en relación con las mujeres y los niños, en este Estado todo sería en común. ${ }^{16}$ Sócrates había omitido intencionalmente este punto, porque sabía qué clase de enjambre de discursos se atraería. Ahora tendría que comenzar como si lo hiciera desde el principio.

$\mathrm{Y}$ de hecho en seguida se exponen los fundamentos de un nuevo Estado: sería posible, y al mismo tiempo útil, que a las mujeres les fueran dadas la misma educación y las mismas tareas (primera ola: 451c4-457b6). El uso del concepto de posibilidad llega a ser claro ya en la primera ola: para mostrar que es posible que les sean dadas a las mujeres las mismas tareas, Sócrates intenta demostrar por medio de la dialéctica que las mujeres, en vista de las tareas de la administración

${ }^{15}$ Véanse las pp. 178-179, supra.

${ }^{16}$ Eso fue en $423 \mathrm{e} 6-424 \mathrm{a} 2$. 
del Estado, tienen la misma naturaleza que los hombres. Al final dice: "No hemos expuesto entonces como ley un imposible o algo semejante a vanos anhelos, puesto que hemos establecido la ley efectivamente en conformidad con la naturaleza" (456b12-c2). La pregunta sobre la posibilidad del Estado ideal se decidirá, pues, mediante la determinación de si el Estado es o no es en conformidad con la naturaleza ( $x \alpha \tau \grave{\alpha}$

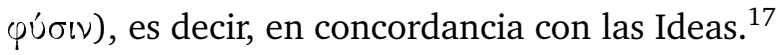

Pues bien, el nuevo Estado caracterizado por la comunidad de mujeres y niños se investiga, en primer lugar, en vista de su utilidad. El máximo bien en el Estado habría de ser su unidad, la cual, en cuanto unidad de placer y dolor, es garantizada de manera óptima por la comunidad de mujeres y niños (segunda ola: 457b7-466d5). Por lo tanto, en este peldaño el Estado ideal es bosquejado primeramente en su unidad relativa al cuerpo.

Y es hasta la tercera ola (466d6-541b5) cuando se trata por fin el tema de su posibilidad. Sócrates incluso había postergado intencionalmente el discurso mediante una muy prolongada digresión acerca de la conducción de la guerra en el Estado ideal, puesto que tenía que reservar sus fuerzas para la más grande y más peligrosa de las tres olas. Peligrosa sería la ola, más precisamente, a causa de que su punto

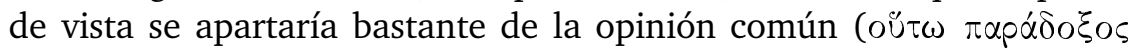
$\lambda o ́ \gamma o \varsigma, 472 a 6$ ). La discrepancia entre el filósofo y la multitud refleja la discrepancia entre las dos tendencias históricas, esto es, entre la filosofía y el pueblo corrompido por los sofistas, entre la posibilidad y la dificultad de realización del Estado ideal, respectivamente. Además de eso, en vista de esa situación ya no tendría que sorprender el hecho de que Platón deje sin mencionar el mismo objeto que, de todos modos, encierra dentro de sí la mención de su doctrina de los principios ante los nada filosóficos interlocutores de las Leyes.

Antes de que busque probar la posibilidad, Sócrates llama la atención primero sobre la diferencia ontológica que por necesidad se da entre un modelo y la realidad (472b3-473b3): en el discurso precedente se trataba de buscar un parádeigma de justicia e injusticia para ver, en vista de este modelo, cómo se comportan ambas en relación con la felicidad. El que fuera entonces más semejante al hombre justo o injusto en la realidad obtendría el destino de justicia o injusticia más semejante. Pero un hombre no debe exigirse que no se diferencie de la justicia misma en el más mínimo grado.

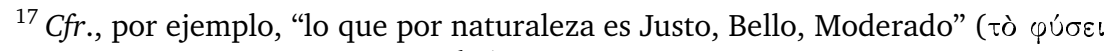

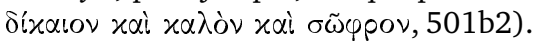

Diánoia, vol. LIV, no. 63 (noviembre 2009). 
Así como, además, la calidad de un cuadro no ha de medirse por el hecho de que un hombre excelentemente bien pintado pueda o no darse después en la realidad, así también habría de evaluarse el parádeigma del buen Estado expuesto por ellos al margen de la posibilidad de su realización.

En segundo lugar, empero, estaría en la naturaleza del hecho que éste alcance menos la verdad que el discurso. Por eso estarían ya satisfechos si llegasen a mostrar que se hubiera instituido un Estado tan se-

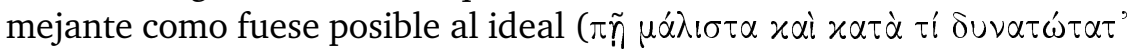

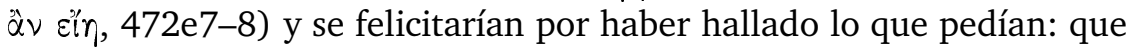
eso pudiera ser real.

En el Estado descrito y en sus cuatro imágenes se trata, entonces, de una Idea y de las privaciones correspondientes a ella, la cual, como la Idea de la Justicia e Injusticia, nunca puede existir en la realidad exactamente como fue pensada. La diferencia es ontológica. El hombre justo no es la Idea de la Justicia, sino que tiene únicamente participación en ella. ${ }^{18}$ Él es definido como aquel que, en analogía con la unidad del cosmos de las Ideas, llega a componerse uno solo de muchos ( $\check{\varepsilon} v \alpha$

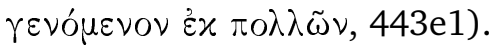

Cuando las Leyes proclaman que no humanos, sino solamente dioses o sus hijos vivían en este Estado refiriéndose al parádeigma, descalifican la naturaleza humana frente a la naturaleza de los dioses y de sus hijos que habitan el Estado ideal, sugieren que la Idea de la Justicia no pertenece al mundo de las Ideas, sino al ámbito de los dioses, a saber, al ámbito del alma cósmica. Esto confirma el discurso segundo de Sócrates en el Fedro, donde los dioses olímpicos representan distintas virtudes éticas en función de Ideas. De este modo, las Ideas éticas estarían en el mismo nivel que las Ideas de artefactos en el libro diez de la República. ${ }^{19}$

La diferencia entre hombres, por un lado, y estas Ideas o dioses, por el otro, la explica Aristóteles en la Metafísica:

Por consiguiente, de tal principio penden el cielo y la naturaleza. Y es como el mejor modo de vida para nosotros durante poco tiempo (pues él siempre es así; para nosotros, en efecto, es imposible), puesto que también

\footnotetext{
${ }^{18}$ De manera semejante: "algún hombre que se halle en equilibrio y consonancia con la excelencia, de palabra y acto, tan perfectamente como sea posible" (a $\alpha \delta \rho \alpha \delta \varepsilon$

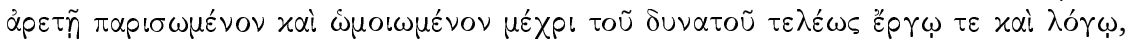
498e3-4).

${ }^{19}$ Cfr. Halfwassen 2000, p. 49, que a su vez refiere a Gaiser 1998.
} 
su actividad es placer (y por eso vigilia, percepción, intelección son lo más placentero, y esperanzas y recuerdos por causa de éstas). ${ }^{20}$

No obstante que la Idea de la Justicia está en el nivel anímico, tiene su modelo en el cosmos de las Ideas. La relación que el hombre tiene con ella corresponde a su relación con el parádeigma del Estado ideal:

[Él,] mirando y contemplando las cosas que están bien dispuestas y se comportan siempre del mismo modo, sin sufrir ni cometer injusticia unas a otras, conservándose todas en orden y conforme a la razón, tal hombre las imita y se asemeja a ellas al máximo. ¿O piensas que hay algún mecanismo por el cual aquel que convive con lo que admira no lo imita? -Es imposible. -Entonces, en cuanto el filósofo convive con lo que es divino y ordenado se vuelve él mismo ordenado y divino, en la medida en que esto es posible al hombre. (500c2-d1)

Por lo tanto, no está permitido suponer aquí una versión débil del concepto de posibilidad en el sentido de que, como lo sostiene Ferber (1991), no sería posible al filósofo ninguna contemplación definitiva de la Idea del Bien. ${ }^{21}$ Está claro que se habla de contemplación de las

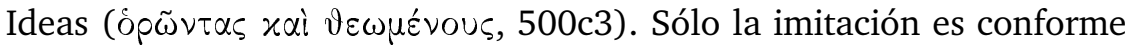
a la manera humana, es decir, no es eterna como lo es para los dioses y las Ideas. ${ }^{22}$ De manera semejante, un Estado que mediante la acción ( $\pi \rho \tilde{a} \xi(\varsigma)$ está sometido al cambio, nunca puede alcanzar una estabilidad igual a la que alcanza la Idea del mismo desplegada en el diálogo.

Es también interesante la comparación con el pintor que, por inspiración divina, esboza un parádeigma (472d5), del cual, como el poeta en Apol. 22b5-c4, no puede dar cuenta alguna. Se trata, entonces, de una

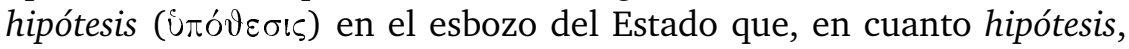
ya tiene estatus de Idea ( $c f r$. Chen 1992, p. 33), pero que requiere una

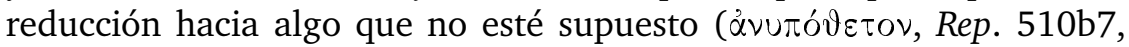
511b6).

Por esa razón, la posibilidad del Estado esbozado no depende en absoluto del grado de su aproximación al parádeigma, sino más bien,

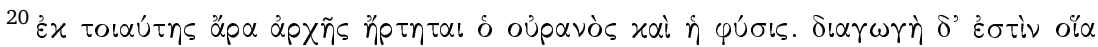

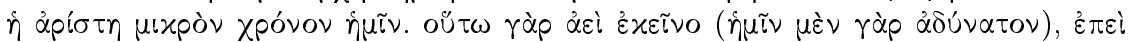

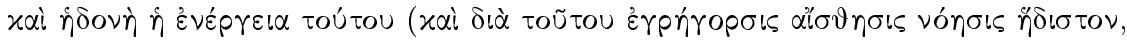

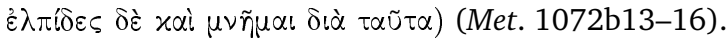

${ }^{21}$ Contra lo que propone Ferber, cfr. Szlezák 1997, y 2003, p. 84.

${ }^{22}$ Con respecto a la consecución de la Idea de lo Bello en el Symposium, cfr. Albert 1989. 
como en la primera ola, de si la Idea del Estado esbozada en el sentido de una hipótesis ( ( $\varkappa \alpha \tau \grave{\alpha} \varphi u ́ \sigma \iota \nu)$ o no; es decir, de si ella se deja reducir hacia la Idea del Bien como causa de todo ser. Aunque en la segunda ola el estatus de Idea presupone ya esto, y la unidad del Estado en forma de comunidad de mujeres y niños era susceptible de ser reducida a la Idea del Bien - en cuanto que el Uno concede a cada uno su unidad-, todavía no se ha dado una reducción dialéctica hacia la Idea del Bien. La posibilidad ya está garantizada, por consiguiente, en forma de una hipótesis, pero debe ser elevada con los medios de la dialéctica hacia una absoluta claridad. Por esa razón, según las reglas del método hipotético, la unidad corporal del Estado ideal debe ser reducida a través de los diferentes peldaños ontológicos hasta el Uno mismo.

La posibilidad de la comunidad de mujeres y niños depende en primer lugar de la unión personal de filósofos y de los que detentan el poder (473b4-e5). Una vez que el filósofo es determinado como aquel que ama la verdad y el Uno en lugar de muchas apariencias (474b3480a13, esp. 479a3-5), la naturaleza ética de su alma se deriva de este parentesco con el Uno (484a1-487a8). Con respecto a esta determinación de la naturaleza filosófica, se refutan dos objeciones opuestas por Adimanto: por un lado, los filósofos pasan por ser inútiles, porque la multitud no sabe usarlos (487c4-489d6); por otro, la mala fama de la filosofía resulta del hecho de que las auténticas naturalezas filosóficas son corrompidas por la multitud y su lugar es ocupado por los sofistas. En las circunstancias actuales, por consiguente, sólo pocos filósofos verdaderos pueden conservar su naturaleza (489d7-497a5). La discusión sobre la posibilidad de una naturaleza filosófica es descrita, según la filosofía de la historia de Platón, como una lucha entre las fuerzas buenas y las malas en la sociedad de Atenas. La multitud y los sofistas ${ }^{23}$ amenazan constantemente el desarrollo de una naturaleza filosófica: en este contexto Sócrates afirma incluso que no sería posible que en esas circunstancias —es decir, bajo el influjo corruptor de la multitud

${ }^{23}$ Por un lado, la multitud es el sofista más grande (492a5-d7); por otro, los sofistas son sirvientes de la multitud (493a6-e1). Ahora bien, el que la democracia extrema y el gobierno tiránico de uno solo sean lo mismo en sustancia (Leyes 693d2-694a1) no es, por esa razón, ninguna novedad en las Leyes (Laks, p. 89). La democracia esbozada en el libro VIII de la República es una democracia mejor, puesto que nada tiene en común con la ilegalidad de la democracia desviada de las Leyes ni con las conductas en Atenas. Con respecto a la compatibilidad de las teorías constitucionales de la República, el Político y la Carta Séptima, cfr. Bruchmüller 2008, pp. 129-133. 
en las asambleas populares de Atenas- se origine un filósofo, y que el solo hecho de emprender en privado la formación de una tal naturaleza sería una necedad ( $\alpha$ voı $\alpha, 492 \mathrm{e} 2$ ) (492d9-e5, 495a2-3). Sin embargo, aun bajo estas circunstancias el género filosófico no puede ser eliminado por completo. Los dioses evidentemente lo impiden (492e6-493a2, 496c3-5, 502a9-b2).

Una vez que se ha probado la posibilidad de una naturaleza filosófica por su misma existencia, es preciso mostrar en un paso subsecuente cómo debe ejercer su poder en el Estado correspondiente. Para ello es necesario que siempre se conserve el mismo concepto del mejor Estado. Esto presupone, sin embargo, una educación perfecta del alma intelectual en los filósofos (497a6-498c4). Por ello, la existencia permanente de filósofos y, por lo tanto, la continuidad del Estado ideal sólo pueden ser garantizadas mediante una educación sistemática del loguistikón (cfr. Szlezák 2003, p. 83).

También este punto, el proyecto entero del Estado ideal, debe ser asegurado contra la multitud en dos pasos. El hecho de que la multitud, en efecto, haya de ser enemiga de los filósofos reside en el hecho de que no sabría a quiénes señalar como filósofos y en que nunca antes había conocido un discurso tan verdadero y natural como el actual. Pero puesto que la multitud está libre de envidia y es apacible (ą

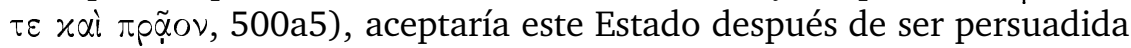
mediante palabras amistosas ( $\pi \alpha \rho \alpha u v \vartheta o u ́ \mu \varepsilon v o \varsigma, 499 \mathrm{e} 2$; 498d6-500e5).

En un segundo paso, Sócrates explica que los filósofos primero purifican el Estado y de qué manera transfieren las Ideas de las virtudes particulares en la realidad. Finalmente, también aquellos que en la promulgación del teorema del rey filósofo en serie cerrada querían arremeter contra él, por vergüenza confesarían ahora que se han apaciguado y

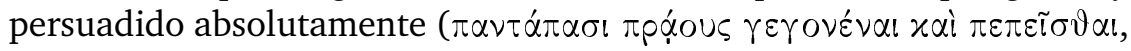
502a1; 500e5-502a3). Ahora incluso Trasímaco, que ocupa el lugar de suplente, es llamado amigo (498c5-d4).

También aquí la orientación opuesta de las distintas tendencias del desarrollo ha quedado representada en el diálogo: la multitud de mejores se deja convencer más fácilmente; los pocos que especialmente muestran mala naturaleza, no obstante, al final también llegan a ser vencidos mediante el recurso a la doctrina de las Ideas. El medio, por consiguiente, no es acaso la violencia, sino la retórica dialéctica. La multitud llega a ser ganada como la instancia de menor valor ontológico para una relación recíproca con los gobernantes filósofos en forma

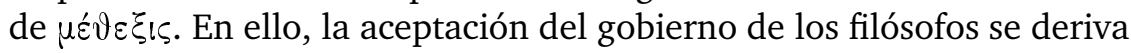
de la naturaleza filosófica. La conducción errática de la multitud que 
hacen los sofistas corresponde a los peligros de una naturaleza filosófica. Como la existencia de la naturaleza filosófica podía ser garantizada por una educación dialéctica, así también la virtud de la multitud se asegura por los medios de la dialéctica. También es notable, pero en completa concordancia con el pensamiento platónico, el hecho de que la multitud sea ganada para algo antes de que esto mismo en general se haya explicado: la educación dialéctica sólo es accesible para quien es apto para ella. ${ }^{24}$

El gobierno de los filósofos, y con él la realización de la comunidad de mujeres y niños, depende entonces de la posibilidad de la virtud ética del individuo; es decir, de su unidad y justicia. ${ }^{25}$ Ésta se ve constantemente amenazada, pero puede garantizarse mediante la educación correcta de la parte racional del alma de los gobernantes filósofos, la cual consiste en la formación gradual como dialéctico y su perfeccionamiento, la cual, por su parte, culmina en la contemplación de la Idea del Bien y aquí completa la unidad de la parte más alta del alma. ${ }^{26}$ No es sino hasta después de que ha tratado la formación de los filósofos que Sócrates explica, a modo de compendio, que la constitución en cuestión y sus instituciones no son anhelos piadosos, sino posibles sólo si los verdaderos filósofos llegan al poder (540d1-e3).

Por lo tanto, los libros intermedios representan un ejemplo del método hipotético. Según Rep. 511b3-c2, debemos imaginarnos el ámbito de lo inteligible como aquello que "la razón misma aprehende, por medio de la facultad dialéctica, y hace de los supuestos no principios sino realmente supuestos, que son como peldaños y trampolines hasta el principio del todo, que es no supuesto". ${ }^{27}$ Estos peldaños y trampolines se pueden observar en el texto mismo: mientras que la primera ola sólo respeta la naturaleza de las mujeres, la segunda ola reconoce el valor de la comunidad de mujeres y niños y traza la Idea de un nuevo Estado en forma de la unidad corporal. La tercera ola, al requerir cierta naturaleza filosófica, inicia con un nuevo peldaño; la fundamentación

\footnotetext{
${ }^{24}$ Con respecto a la naturaleza esotérica del esbozo del Estado ideal, $c f r$. también Szlezák 2003, pp. 85-86.

${ }^{25} \mathrm{La}$ unidad de la naturaleza ética expresada en el término de la mesura ( $\varepsilon \mu \mu \varepsilon \tau$ í $\alpha 386 \mathrm{~d} 7,8)$ se deduce del amor a la unidad de la Idea en oposición a las muchas apariencias (476a4-7, 485a10-b3).

${ }^{26}$ En relación con los pasos de la formación del loguistikón, cfr. Bruchmüller 2008, pp. 213-218.

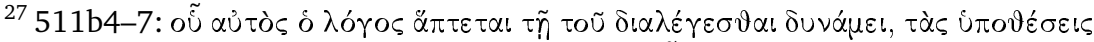

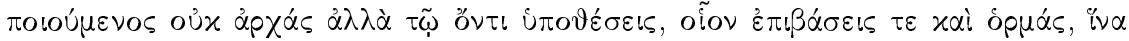

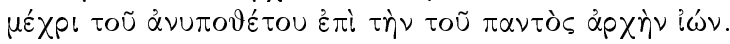


de esta naturaleza en la educación dialéctica marca expresamente un

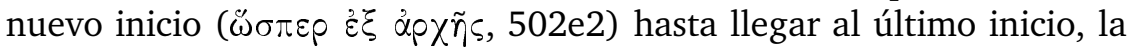
Idea del Bien. Resumiendo, pues, la unidad corporal es reducida a la unidad anímica, y ésta a la unidad de las Ideas. Con ello se prueba perfectamente el estatus de Idea del Estado ideal. El Estado es en con-

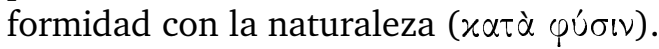

Tal como en el Timeo, donde el cuerpo del universo no puede darse sin el alma cósmica (34b10-35a1) ni ésta sin el demiurgo, el cual debe entenderse como totalidad del cosmos de las Ideas ( $c f r$. Halfwassen 2000), así la unidad corporal del Estado postulada en la segunda ola tampoco es posible sin la unidad psíquica del mismo garantizada mediante la naturaleza filosófica, la cual, a su vez, sólo puede darse mediante el conocimiento del Bien de los gobernantes filósofos. Tal como en el Timeo, también aquí la secuencia de las conversaciones es acentuada de manera dramática. ${ }^{28}$ Puesto que el cosmos sensible es una imagen del cosmos de las Ideas, una Idea que no es realizable sería en el pensamiento platónico una inconsistencia extraña y, además, filológicamente no demostrable. En el momento en que Platón eleva su Estado ideal a la Idea, él debe creer en la posibilidad de su realización. En este sentido, Platón no está alejado de la realidad, sino que sólo es filosóficamente consecuente.

Después de la fundamentación del Estado ideal en la Idea del Bien, se emprende de nueva cuenta el tema de su implementación. Ahora se realiza la purificación antes sólo mencionada ${ }^{29}$ con el envío de todos los habitantes mayores de diez años al campo, de modo que las condiciones ideales para una educación exitosa estén dadas. De este modo el Estado llegará a ser feliz de la manera más rápida y fácil (540e5541b5). Puesto que la comunidad de mujeres y niños sólo concernía a los dos primeros estamentos, debe entenderse que los adultos simplemente son expulsados al tercer Estado, el cual en su mayor parte es por naturaleza activo en el campo.

De manera semejante, aún hoy en día muchos padres viven separados de sus hijos para que estos últimos puedan disfrutar de una buena educación. Puesto que el pueblo fue persuadido antes, mediante la retórica dialéctica, de aceptar el gobierno de los filósofos, no se puede entender por qué estas medidas habrían sido eliminadas en las Leyes a causa de su carácter no "humano" y la imposibilidad de realizarlas, como piensa Laks (pp. 77-78).

${ }^{28}$ Cfr. 457e7-458b8.

${ }^{29}$ Véase la p. 186, supra.

Diánoia, vol. LIV, no. 63 (noviembre 2009). 
Finalmente, después de que también se trató acerca de las constituciones injustas y fueron sopesadas entre sí las ventajas y las desventajas de la justicia y la injusticia, Sócrates afirma que el hombre razonable habría de esforzarse en la política sólo en su propio Estado, pero no en su patria. Si bien, como cree Glaucón, no existe un Estado como el ideal sobre la tierra - pues no depende de eso-, sí existiría en el cielo un parádeigma de él, con vistas al cual habría de organizarse el que quiera verlo (592a5-b6). De nuevo se confirma: Platón sabía que en la praxis el Estado era tanto como no realizable: pero de ello no depende para nada la posibilidad de su realización. Lo esencial es la existencia de la Idea de la Justicia, con vistas a la cual por lo menos el filósofo solitario se organiza y a la cual Sócrates no cesaría de defender mientras pudiese respirar (368b7-c2).

\section{III.2. LA CARTA SÉPTIMA}

Laks (p. 103) aprecia correctamente que los sucesos en Siracusa no fueron apropiados para que Platón tuviera esperanzas en la realización del Estado ideal y que por eso tampoco podía aprender de esa experiencia. En efecto, cuando en su primer viaje Platón habló por primera vez con Dión sobre el contenido de la República, no sabía en absoluto que, sin notarlo, activaba la disolución de una tiranía (Ep. VII 327a1-5). En los pasajes posteriores llega a ser claro entonces que Platón no tenía ninguna esperanza en sus dos viajes (327e2-328b1, 337e3-340b1). Antes bien, acentuó sus tareas en la Academia (329b1-3), las cuales son, con mucho, más comparables al proyecto del Estado ideal.

Es altamente probable que, no obstante, el desarrollo de los acontecimientos en Siracusa dio ocasión a la redacción de las Leyes. Tras el destierro de Dionisio y la erección de un Consejo por Dión se requirieron nuevas leyes; sin embargo, Platón tampoco podía permitirse entonces abrigar ninguna esperanza en que los siracusanos aprovecharan bien su obra legislativa. Aunque Dión quería actuar según el consejo de Platón, estaba lejos de disponer del poder necesario para ello (cfr. Szlezák inédito).

Por consiguiente, las circunstancias no obligan a suponer que Platón haya escrito las Leyes porque hubiese entendido que el Estado ideal de la República de ningún modo era realizable. Así como nunca abandonó la idea de que un Estado de tal clase era posible, en esos años intermedios tampoco creyó que fuera fácil su realización. Puesto que la República esboza la Idea del mejor Estado y las Leyes presentan un bosquejo de un Estado más fácil de realizar, ambas obras tienen una función completamente diferente y no se excluyen una a la otra. 


\section{III.3 LAS LEYES}

Estos resultados concuerdan con la mención del esbozo del Estado ideal en el libro V de las Leyes (739a1-740a2). Antes de que el Ateniense pueda conceder leyes sobre las relaciones de propiedad, debe esclarecer qué clase de constitución tiene en mente. En efecto, lo mejor sería proponer una constitución óptima, una mejor en segundo grado y otra mejor en tercer grado, para confiar luego el derecho de elección a aquel que decide acerca del establecimiento de una constitución.

En el mejor de los Estados todas las cosas han de ser establecidas de modo que todo, hasta donde sea posible, según el proverbio, sea común a los amigos. No sólo las mujeres, los niños y las propiedades habrían de ser comunes en tal Estado, sino que incluso las partes del cuerpo habrían de dar la impresión de que trabajan en común. Más allá de esto, el elogio y el reproche, tanto como el placer y el dolor deben ser comunes a todos, de modo que todas las medidas tiendan a cumplir la finalidad de producir la unidad máxima posible en el Estado. Nada puede concebirse como más correcto y mejor que lo que haya en este Estado. Por consiguiente, habría de imaginarse un Estado en el que habitan dioses e hijos de dioses; o sea, más de uno. Un Estado de tal clase debe contemplarse como parádeigma de una constitución y ser puesto en práctica hasta donde sea posible.

El Estado esbozado ahora, por el contrario, podría seguirle quizá como más próximo al Estado inmortal y tomar el segundo rango. El tercer Estado, en cambio, debe ser tratado después.

En el Estado de las Leyes, por consiguiente, a causa de la actual generación, instrucción y formación, los habitantes no deben dedicarse en común a la agricultura.

En relación con la posible realización del Estado ideal, el texto es bastante claro: se invita expresamente al lector a que elija de entre las tres constituciones la más adecuada a su fin. ¿Tendría sentido esto si alguna de las constituciones en principio fuera imposible? En relación con la primera constitución se dice: "sea que esto se dé ahora en algún

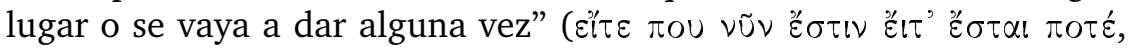
739c3). Entonces, si se dice que en ella sólo habitan dioses e hijos de dioses, no se trata de una contradicción o de una corrección. En su manera de existir como parádeigma (739e1) pueden habitar efectivamente en ella sólo seres inmortales. Bajo la constitución inmortal que cada Estado debe imitar se entiende la Idea inmutable de la que también se habló en la República. ${ }^{30}$ Nada impide, sin embargo, que también

${ }^{30}$ Véanse las pp. 182-185, supra. 


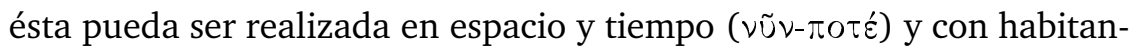
tes mortales. Si bien el Ateniense supone entonces que no es sino la segunda constitución la que sigue al arquetipo de la manera más próxima, esto no expone de nuevo una contradicción dado que expresa aquí el pensamiento de manera tan cautelosa en optativo con el adicional $\pi \omega ́ s(739 e 4)$ y no quisiera hacer un juicio general. ${ }^{31}$ Lo que él quisiera decir, sin embargo, es que para la fundación de la colonia en Magnesia el primer Estado está excluido. Un poco después sigue también la fundamentación: la población no fue engendrada, criada ni educada

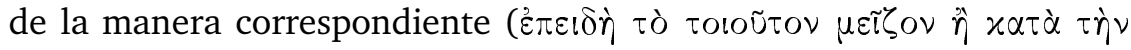

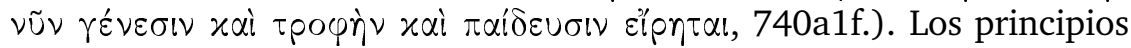
de educación tratados previamente no pueden ocupar el lugar del programa de educación detallado y difícil de la República y, por lo tanto, tampoco pueden realizar el primer Estado. ${ }^{32}$ Pero de ningún modo se excluye la posibilidad de otro engendramiento y otra educación, y por eso existe la posibilidad del primer Estado.

Esto, en efecto, tiene como consecuencia que, al contrario de la hipótesis sobre el desarrollo, se pone en cuestión no la posibilidad del Estado ideal, sino la del segundo Estado fundado sólo en la doctrina del alma ${ }^{33}$ (960b5-969d3). Tal como sucede en la República, también

${ }^{31}$ A manera de ejemplo, en 965e2-4 quedará claro cuánta consideración tiene el Ateniense por el nivel de sus interlocutores.

${ }^{32}$ Otros pasajes tampoco descalifican al Estado ideal habitado solamente por dioses frente al Estado de las Leyes (Laks, p. 76). Bajo los llamados "antiguos legisladores, que lo hacían para los héroes, hijos de dioses, [...] y siendo ellos mismos progenie de dioses, daban leyes para otros nacidos de los mismos" (oi $\pi \alpha \lambda a$ เoi vo-

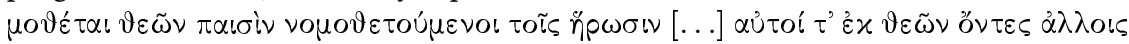

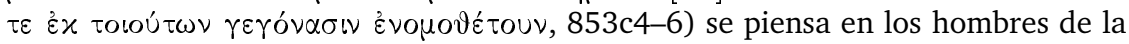
edad de oro (Leyes 712e9-714a8), los cuales viven una vida dichosa bajo la suprasupervisión de Cronos. Que ellos disponen del arte de la legislación se expone de manera metafórica, como en Filebo 16c5-10.

Además, 875c3-d6 también apoya mi interpretación, donde se supone un hombre que, al igual que los filósofos en la República (véanse las pp. 185-186, supra)

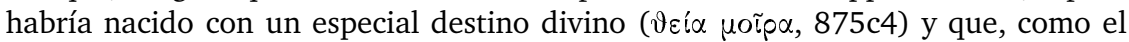
hombre de Estado en el Político (véanse las pp. 179-180, supra), no está sujeto a lo que dispone la ley, porque obedece en todo al nous. Como en la República, se duda ciertamente de la existencia de tales hombres; no obstante, su posibilidad

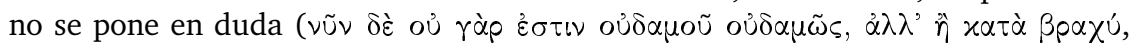
$875 \mathrm{~d} 2-3)$.

33 "Pero me parece que eso es lo que aparentemente todavía falta a nuestras leyes, cómo debe llegar a darse en ellas naturalmente esa inmutabilidad. $\mathrm{Cl}$. —No mencionas algo de poca monta, si en realidad no es posible descubrir cómo podría llegar a tener uno una posesión semejante para cada cosa. At. - Sin embargo es 
aquí el Estado mismo representa al cuerpo. La asamblea nocturna, por el contrario, se divide entre los más jóvenes y los ancianos, los cuales son comparados con los ojos y el intelecto de un individuo (964e1965a4). Su más importante tarea es el conocimiento de la causa de la unidad de la virtud, la cual es el fin del Estado que ha de preser-

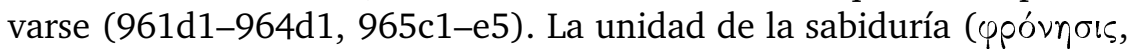
965d2) se funda, no obstante, en la unidad y en la totalidad del cosmos de las Ideas, la cual se debe investigar en su relación con la Idea del Bien, con el Uno mismo. ${ }^{34}$

Pero para que se acepten las leyes de la educación de los miembros de la asamblea nocturna es necesaria una instrucción realizada me-

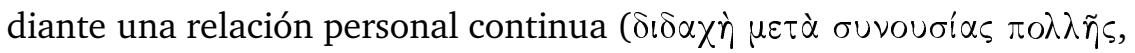
968c6). Por eso, los contenidos pertinentes no son impronunciables ( $\alpha$ rópp $\tau \tau)$, ciertamente, pero no se permite pronunciarlos anticipada-

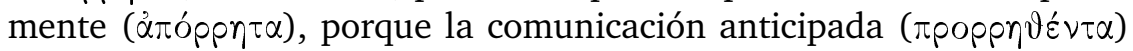
no pondría de manifiesto ninguna de las cosas de que se habla $(968 \mathrm{e} 2-$ 5). ${ }^{35}$ La caracterización de este saber corresponde a la del saber de las Ideas en la crítica a la escritura del Fedro y la Carta Séptima. Por eso, la constitución política entera habría de estar cimentada sobre arena y su realización abandonada al azar. No obstante, el Ateniense se declara dispuesto a mantener esta relación personal continua con Clinias y Megilo, para que los miembros de la asamblea nocturna posean la educación necesaria y la constitución, que se asemeja a un sueño, pueda ser hecha realidad (968e7-969d3). Por lo tanto, el bosquejo de las Leyes al final también está fundado, como el Estado ideal de la República, en el saber de los principios últimos del dialéctico representado por el Ateniense. Pero, puesto que aquí el saber de las Ideas únicamente se introduce para la instauración de la virtud y de la unidad del Estado y, al contrario de la República, dado que no existe en el Estado mismo, es importado del exterior, sólo se trata del segundo mejor de los Estados.

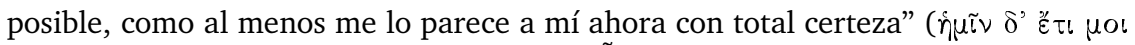

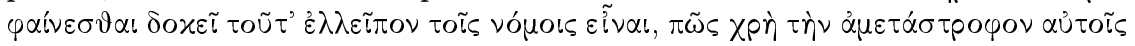

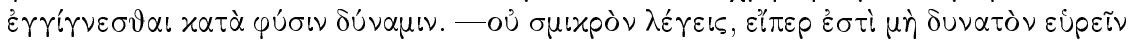

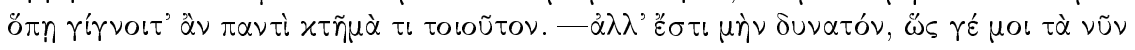

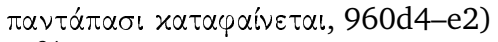

34 “[D]ecir de forma adecuada qué es hacia lo que hay que mirar, ya sea como uno, como conjunto o como ambas cosas o sea como fuere por naturaleza." ( $\alpha \mathrm{\nu}$

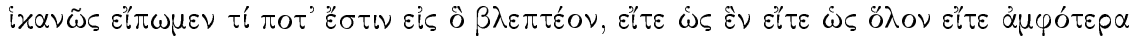

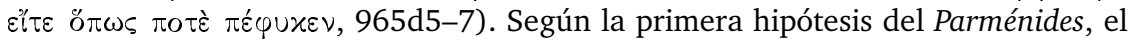
Uno ya no es un todo.

${ }^{35}$ Para el significado del juego de palabras, cfr. Szlezák 2004, p. 51. 
Puesto que Platón suponía tres campos ontológicos - las apariencias, el alma y las Ideas- pero fundó el primer Estado en la suposición de las Ideas, y el segundo en la hipótesis del nous, es preciso suponer que el tercer Estado hubiese tomado como referencia al mundo sensible. Las tres constituciones son ontológicamente posibles y no se excluyen entre ellas.*

\section{BIBLIOGRAFÍA}

Obras de Aristóteles y Platón

Aristóteles, 1950, Aristotelis Physica, ed. W.D. Ross, Clarendon, Oxford. —, 1957, Aristotelis Metaphysica, ed. W. Jaeger, Clarendon, Oxford. Platón, 1995, Platonis opera, ed. E.A. Duke, W.F. Hicken, W.S.M. Nicoll, D.B. Robinson, J.C.G. Strachan, Clarendon, Oxford.

— $1900-1907$, Platonis opera I-V, ed. J. Burnet, Clarendon, Oxford.

—, 2008 , Platón. Diálogos: IV República, trad., introd. y notas C. Eggers Lan, Gredos, Madrid (Biblioteca Clásica Gredos).

—_, 2008, Platón. Diálogos: V Parménides, Teeteto, Sofista, Político, trad., introd. y notas M.I. Santa Cruz, Á. Vallejo Campos y N.L. Cordero, Gredos, Madrid (Biblioteca Clásica Gredos).

—_, 2008, Platón. Diálogos: VII-IX Las Leyes, trad., introd. y notas F. Lisi, Gredos, Madrid (Biblioteca Clásica Gredos).

\section{Bibliografía secundaria}

Albert, K., 1989, Über Platons Begriff der Philosophie, Academia Verlag, Sankt Augustin (Beiträge zur Philosophie, 1).

Bruchmüller, U., 2008, Die Lebensperioden von Kosmos, Polis und Individuum in der Philosophie Platons: Zur platonischen Seelenlehre in ihren ontologischen Voraussetzungen, Stockholm University, Estocolmo.

Chen, L., 1992, Acquiring Knowledge of the Ideas. A Study of the Methods in Plato's Phaedo, Symposium and Republic, Franz Steiner, Stuttgart (Palingenesia, 35).

*El presente ensayo fue redactado en el marco de mi proyecto "Platons Phaidros und Politeia. Platons Rhetorik in ihren ontologischen Voraussetzungen", en el Instituto de Investigaciones Filológicas de la UNAM con la colaboración de la Coordinación de Humanidades. Quiero dejar aquí constancia de mi gran agradecimiento a ambas instituciones; asimismo, aprovecho para expresar mi profunda gratitud al doctor Jesús Araiza por su competente y cuidadosa labor en la traducción. Las citas textuales de la República en lengua castellana proceden de la versión de Conrado Eggers Lan; las citas del Político, de la de María Isabel Santa Cruz, y las de las Leyes, de la de Francisco Lisi. Agradezco mucho, por lo demás, las sugerencias de los dos dictaminadores anónimos. 
Cherniss, H., 1945, The Riddle of the Early Academy, University of California Press, Berkeley.

_ 1944, Aristotle's Criticism of Plato and the Academy, Johns Hopkins Press, Baltimore.

Ferber, R., 1991, Die Unwissenheit des Philosophen oder Warum hat Platon die 'ungeschriebene Lehre' nicht geschrieben?, Academia Verlag, Sankt Augustin (Academia Philosophical Studies, 4).

Gaiser, K., 1998 [1963], 3a. ed., Platons ungeschriebene Lehre. Studien zur systematischen und geschichtlichen Begründung der Wissenschaften in der Platonischen Schule, E. Klett, Stuttgart.

Görgemanns, H., 1960, Beiträge zur Interpretation von Platons Nomoi, C.H. Beck, Munich.

Guthrie, W.K.Ch., 1975, A History of Greek Philosophy, Vol. IV: Plato: The Man and his Dialogues, Earlier Period, Cambridge Univesity Press, Cambridge.

Halfwassen, J., 2000, "Der Demiurg: Seine Stellung in der Philosophie Platons und seine Deutung im antiken Platonismus", en A. Neschke-Hentschke (comp.), Le Timée de Platon. Conributions à l'histoire de sa reception, Peeters, Lovaina/París (Bibliothèque Philosophique de Louvain, 53), pp. 39-62.

Hermann, K.F., 1839, Geschichte und System der platonischen Philosophie, C.F. Winter, Heidelberg.

Kahn, Ch.H., 1996, Plato and the Socratic Dialogue. The Philosophical Use of a Literary Form, Cambridge University Press, Cambridge.

— 1993, "Proleptic Composition in the Republic, or Why Book I Was Never a Separate Dialogue", Classical Quarterly, vol. 43, no. 1, pp. 131-142.

Krämer, H.J., 1959, Arete bei Platon und Aristoteles. Zum Wesen und zur Geschichte der platonischen Ontologie, C. Winter, Heidelberg (Abhandlungen der Heidelberger Akademie der Wissenschaften, philosophisch-historisch Klasse, 6).

Laks, A., 2007, La filosofía política de Platón a la luz de las Leyes, trad. Nicole Ooms, Centro Peninsular en Humanidades y Ciencias Sociales/Instituto de Investigaciones Filológicas, UNAM, Mérida (Didaskalía, 1).

Reale, G., 1997 [1984], 20a. ed., Per una nuova interpretazione di Platone. Rilettura della metafisica dei grandi dialoghi alla luce delle "Dottrine non scritte", Vita e Pensiero, Milán.

Schleiermacher, F., 1804, Platons Werke, vol. I. 1, Realschulbuchhandlung, Berlín.

Schöpsdau, K., 1994, Platon. Nomoi, Übersetzung und Kommentar, vol. 1, Vandenhoeck und Ruprecht, Gotinga.

Szlezák, Th.A., inédito, "Platon in Syrakus: Politik, Philosophie und Eros", Perspektiven der Philosophie, 2010.

__ 2004, Das Bild des Dialektikers in Platons Späten Dialogen. Platon und die Schriftlichkeit der Philosophie, Teil II, Walter de Gruyter, Berlín/Nueva York.

—_, 2003, Die Idee des Guten in Platons Politeia. Beobachtungen zu den mittleren Büchern, Academia Verlag, Sankt Augustin (Lecturae Platonis, 3). 
Szlezák, Th.A., 1997, recensión sobre Rafael Ferber, "Die Unwissenheit des Philosophen oder Warum hat Platon die 'ungeschriebene Lehre' nicht geschrieben?", Gnomon, vol. 68, pp. 404-411.

—_, 1985, Platon und die Schriftlichkeit der Philosophie. Interpretationen zu den frühen und mittleren Dialogen, Walter de Gruyter, Berlín/Nueva York.

_ 1984, "Aufbau und Handlung der platonischen Politeia", Antike und Abendland, vol. 30, no. 1, pp. 38-46.

Thesleff, H., 1982, Studies in Platonic Chronology, Societas Scientiarum Fennica, Helsinki (Commentationes Humanarum Litterarum, 70).

Vlastos, G., 1991, Socrates. Ironist and Moral Philosopher, Cornell University Press, Ithaca.

Recibido el 14 de abril de 2009; aceptado el 15 de septiembre de 2009. 